\title{
SÍNDROME DE EVANS Y EMBARAZO EN LA ADOLESCENCIA: REPORTE DE UN CASO Y REVISIÓN DE LA LITERATURA
}

\section{Evans Syndrome and teenage pregnancy: case report and review of the literature}

David Andrés Viveros-Carreño, $M D^{1}$; Arturo José Parada-Baños, $M D^{2}$

Recibido: octubre 26/15 - Aceptado: junio 9/16

\section{RESUMEN}

Objetivo: presentar el caso de una gestante adolescente con síndrome de Evans, y hacer una revisión de la literatura respecto a su tratamiento y pronóstico durante el embarazo.

Materiales y métodos: se presenta el caso de una paciente adolescente embarazada con síndrome de Evans, manejada en nuestra unidad, ubicada en un hospital de segundo nivel de referencia en Bogotá (Colombia); se describe la historia clínica, su diagnóstico, manejo y desenlace, y se realiza una revisión de la literatura con énfasis en su tratamiento y pronóstico. Se realizó una búsqueda de literatura utilizando las bases de datos Medline vía PubMed, Embase y la Biblioteca Cochrane a mayo de 2016. Las palabras clave utilizadas fueron "anemia hemolítica autoinmune", "trombocitopenia”, "síndrome de Evans" y "embarazo", en español o inglés, sin límite por año de publicación.

1 Médico; Residente II año, especialidad Obstetricia y Ginecología, Universidad Nacional de Colombia, Bogotá (Colombia). daviveros@ unal.edu.co

2 Médico, ginecoobstetra, Universidad Nacional de Colombia; profesor asociado, Facultad de Medicina, Departamento de Obstetricia y Ginecología, Universidad Nacional de Colombia, Bogotá (Colombia). ajparadab@unal.edu.co
Resultados: se encontraron 79 publicaciones en la búsqueda en Medline y 61 en Embase. De estas, 13 estudios estaban directamente relacionados con el tema. Uno de los artículos corresponde a una revisión sistemática de la literatura y los demás a reportes de caso. Todos los reportes de caso encontrados están incluidos en la revisión sistemática. El síndrome de Evans se debe sospechar cuando se presenta trombocitopenia y hemólisis en la mujer gestante. La patología tiene un curso variable durante el embarazo y amerita un control estricto materno-fetal. Se dispone de alternativas que incluyen el uso de corticoides, gamaglobulina intravenosa y, en algunos casos, el manejo quirúrgico con esplenectomía.

Conclusiones: el síndrome de Evans es una patología rara durante la gestación, se requieren más estudios respecto al tratamiento y pronóstico de la enfermedad que permitan guiar su manejo.

Palabras clave: anemia hemolítica autoinmune, trombocitopenia, embarazo.

\section{ABSTRACT}

Objective: To present the case of a pregnant teenage girl with Evans Syndrome, and to conduct a review of the literature regarding treatment and prognosis during pregnancy. 
Materials and methods: Case presentation of a pregnant teenage girl with Evans Syndrome managed at our unit in a Level II referral hospital in Bogota, Colombia; description of the clinical history, diagnosis, management and outcome; and review of the literature focusing on treatment and prognosis. A search of the literature was conducted using the Medline database through PubMed, EMBASE and the Cochrane library up to May 2016. The key terms used were "autoimmune haemolytic anaemia", "thrombocytopenia", "Evans Syndrome" and "pregnancy", both in Spanish and English, with no restriction by year of publication.

Results: Overall, 79 publications were found in Medline and 61 in EMBASE. Of these, 13 studies related directly to the topic, one was a systematic review of the literature, and the rest were case reports. All the case reports found are included in the systematic review. Evans Syndrome must be suspected when there is thrombocytopenia and haemolysis in the pregnant woman. The course of the disease varies during pregnancy and warrants close maternal and foetal follow-up. Treatment options are available, including steroids, intravenous gamma globulin and, in certain cases, surgical management with splenectomy.

Conclusions: Evans Syndrome is a rare disease during pregnancy. Further studies are needed regarding the treatment and prognosis of the disease in order to guide treatment.

Key words: Autoimmune haemolytic anaemia, thrombocytopenia, pregnancy.

\section{INTRODUCCIÓN}

El síndrome de Evans (SE) es una enfermedad autoinmune poco frecuente, descrita por primera vez en 1951 como el desarrollo simultáneo o secuencial de anemia hemolítica y trombocitopenia de origen autoinmune en ausencia de otra causa subyacente (1).

El síndrome se presenta en niños y adultos, y es más común en mujeres que en hombres, con una relación 2:1. En pacientes gestantes, la tromboci- topenia autoinmune se presenta en 1 a 5 casos por 1.000 embarazos y compromete cerca del $14 \%$ de los recién nacidos, pero hay muy pocos casos reportados de pacientes gestantes con síndrome de Evans (2).

La aparición de la enfermedad se puede dar en un contexto aislado o en asociación con otras enfermedades autoinmunes que incluyen lupus eritematoso sistémico y síndrome antifosfolípidos, síndromes mielodisplásicos, linfoma no Hodgkin, o puede ser inducido por medicamentos (levodopa, cefalosporinas, penicilina, quinidina y algunos AINES) $(3,4)$.

El diagnóstico se basa en los hallazgos de laboratorio, con evidencia de anemia y trombocitopenia, alteraciones en el frotis de sangre periférica, conteo aumentado de reticulocitos e hiperbilirrubinemia indirecta por la hemólisis. Habitualmente, el test de Coombs directo es positivo, tanto para IgG como para complemento C3d, y en ocasiones el test de Coombs indirecto también puede ser positivo (5).

Actualmente se reconoce que el síndrome tiene un espectro amplio, presentándose casos con neutropenia, incluso pancitopenia y, en algunas ocasiones, disminución en los niveles séricos de inmunoglobulinas IgG, IgM e IgA (6). El diagnóstico de laboratorio se basa en la detección de anticuerpos. En el 60 a $80 \%$ de las pacientes con anemia los autoanticuerpos son calientes, es decir, son reactivos a temperatura entre 35 a $40{ }^{\circ} \mathrm{C}$ (7).

La presentación del síndrome de Evans en el embarazo no es frecuente, y menos en adolescentes embarazadas. En el diagnóstico diferencial se deben incluir condiciones que producen trombocitopenia durante el embarazo como el síndrome HELLP y los trastornos microangiopáticos, que tienen un manejo y pronóstico diferente para la madre y el feto (8).

El objetivo de este artículo es reportar el caso de una gestante adolescente con síndrome de Evans adquirido durante la adolescencia y hacer una revisión del tratamiento y pronóstico de esta entidad durante la gestación. 


\section{REPORTE DE CASO}

Paciente de 16 años, estudiante, primigestante, atendida en la consulta de control prenatal de alto riesgo del Hospital de Engativá de Bogotá, institución pública de atención general de mediana complejidad, con un embarazo de 20 semanas por ecografía del primer trimestre. Antecedente de síndrome de Evans diagnosticado hace 4 años, sin antecedentes familiares de importancia. Consumo de sustancias psicoactivas desde los 13 años de edad.

El SE fue diagnosticado a los 12 años, debutó con sangrado abundante durante la menarquia, que ameritó su hospitalización. presentó en ese momento anemia hemolítica autoinmune y trombocitopenia inmune de manera concomitante, con laboratorios: hemoglobina $7,5 \mathrm{~g} / \mathrm{dL}$, hematocrito $23 \%$, recuento de plaquetas de $12.000 / \mu \mathrm{L}$, leucocitos (WBC) de $8.760 / \mu \mathrm{L}$ y neutrófilos $4.230 / \mu \mathrm{L}$, reticulocitos de $4 \%$, Coombs directo positivo fraccionado $\mathrm{IgG}$, complemento normal y $\mathrm{C} 3 \mathrm{~b}$ negativo. Perfil infeccioso negativo para citomegalovirus, toxoplasmosis, Epstein Barr, hepatitis B, hepatitis C, herpes virus y VIH. Aspirado de biopsia de médula ósea normal. Tratada con prednisolona 50 mg/día, transfusión de glóbulos rojos empaquetados y plaquetas.

Presentó dos recaídas, a los 14 y a los 15 años de edad, en ambos casos cursó con anemia y trombocitopenia concomitante, con conteo leucocitario normal; fue tratada con prednisolona $50 \mathrm{mg} / \mathrm{día}$ con recuperación completa. En la segunda recaída se encontraron anticuerpos antinucleares en patrón moteado positivos, y al considerar el riesgo de lupus eritematoso sistémico se adiciona a la terapia cloroquina, en dosis de $250 \mathrm{mg}$ /día, con una adecuada respuesta y sin requerir esplenectomía. La paciente queda en embarazo y suspende voluntariamente la terapia al igual que el consumo de psicoactivos.

$\mathrm{Al}$ ingreso a los controles prenatales, en los laboratorios no hay evidencia de anemia o trombocitopenia (Hb: $14 \mathrm{gr} / \mathrm{dL}$; Hto: $41 \%$, recuento de plaquetas 260.000/ $\mu \mathrm{L}$ ), Coombs directo negativo, Coombs indirecto negativo y frotis de sangre periférica normal. Sin esplenomegalia por ultrasono- grafía abdominal. Debido a la normalidad clínica y paraclínica no se reinician medicamentos.

El embarazo presenta un curso satisfactorio con ganancia de peso adecuada. Se realizan perfiles paraclínicos en búsqueda de hemólisis en las semanas 20 y 32, los cuales son negativos. En la semana 35 de gestación presenta petequias en miembros superiores y descenso del conteo manual plaquetario, aunque en rango normal (152.000/ $\mu \mathrm{L}$; Hb: 14,4; Hto: $43 \%$ ), se inicia prednisolona a dosis bajas de 10 $\mathrm{mg} /$ día con adecuada respuesta clínica e incremento del conteo plaquetario a 223.000/ $\mu \mathrm{L}$. Seguimiento fetal con doppler de arteria cerebral media (ACM) con velocidad pico sistólica normal.

La paciente se hospitaliza en la semana 40 de gestación con inicio espontáneo del trabajo de parto y adecuado bienestar fetal. Atención del parto vaginal eutócico, con recién nacido que pesó 2.770 g, sin signos de anemia o trombocitopenia; laboratorios del recién nacido dentro de límites normales y con Coombs indirecto negativo.

Puerperio inmediato normal, sin hemorragia posparto; seguimiento a los 2 y 4 meses de posparto normal, sin manejo farmacológico.

\section{MATERIALES Y MÉTODOS}

Se realizó una búsqueda de la literatura utilizando las bases de datos Medline vía PubMed, Embase y la Biblioteca Cochrane, a mayo de 2016. Las palabras clave utilizadas fueron "anemia hemolítica autoinmune", "trombocitopenia", "síndrome de Evans" y "embarazo", en inglés o español, sin límite por año de publicación.

Aspectos éticos. Se solicitó autorización a la paciente para la publicación del caso clínico. Se tomaron las precauciones necesarias para garantizar la confidencialidad de la información y el anonimato de la paciente.

\section{RESULTADOS}

Se encontraron 79 títulos en la búsqueda en Medline y 61 en Embase, no se encontró ninguno en la Biblioteca Cochrane. De estos, 13 estaban di- 
rectamente relacionados con el tema; todos los estudios encontrados en Embase estaban incluidos en Medline. Finalmente, se incluyeron 8 estudios: uno corresponde a una revisión sistemática de la literatura (6), que además reporta dos casos, y seis hacen referencia a reportes de caso (9-14); otro es el reporte de dos gestaciones en una misma paciente que se analizan como dos casos (15). Cinco estudios no se incluyeron en la revisión, cuatro de ellos por estar escritos en un idioma diferente español o inglés (16-19), y uno más porque no fue posible obtener el artículo original (20). En total se evalúa la información obtenida para 11 gestaciones.

Ninguna de las pacientes de los casos reportados era adolescente, y las edades fluctuaron entre los 19 y los 34 años, con una media de 24,4 años. Seis pacientes cursaban con su primera gestación (6, 11-15), una con la tercera (10), una con la cuarta (16), una con la quinta gestación (6), y para una de las pacientes no hay información al respecto (9). Se informó la segunda gestación de una de las pacientes reportadas inicialmente como primigrávida (15). No hay reportes de gestaciones múltiples.

Respecto al momento del diagnóstico en el embarazo, en tres pacientes se realizó en el primer trimestre de la gestación (9-11), siendo la octava semana el momento más temprano del diagnóstico (9), en una paciente con el antecedente de la enfermedad ya diagnosticada; en cuatro pacientes se hizo el diagnóstico en el segundo trimestre (6, $14,16)$ y en las restantes en el tercer trimestre; la más tardía fue en la semana 38 (16); en ninguno de los casos se reportó el diagnóstico en el puerperio. En la paciente con reporte de dos gestaciones, en la segunda el test de Coombs fue positivo en el primer trimestre pero luego fue negativo en el seguimiento (15).

Tres pacientes no presentaron síntomas al momento del diagnóstico y este se realizó por el antecedente (9) o por los hallazgos paraclínicos (anemia, trombocitopenia) en dos casos $(14,15)$; cinco pacientes presentaron sangrado clínico que incluyó epistaxis, sangrado gastrointestinal o hema- turia $(10-13,16)$. Una paciente debutó con ictericia en el primer trimestre de la gestación (15), y de las pacientes reportadas por Lefkou (6), una presentó síndrome febril y pancitopenia y otra preeclampsia.

Tratamiento. Para el manejo farmacológico de la enfermedad se han descrito múltiples opciones, sin embargo, se deberá tener en cuenta no solo la efectividad sino también la seguridad para la madre y el feto.

Medicamentos utilizados. En los reportes de la literatura la elección para el manejo farmacológico durante el embarazo son los corticoides, que fueron utilizados en 10 de las 11 gestaciones reportadas (90\%). El único caso en el que no se reportó el uso de corticoides en algún momento del manejo fue en la segunda gestación del caso reportado por Letts (15). La metilprednisolona fue el medicamento elegido en todos los casos, bien como primera elección o como tratamiento complementario y con dosis variables. Fue el único tratamiento administrado en 5 casos $(9-11,14,15)$.

Tres pacientes recibieron gamaglobulina endovenosa $(6,12,16)$, una paciente recibió hidroxicloroquina y azatioprina como parte de su terapia (6) $\mathrm{y}$ dos pacientes fueron esplenectomizadas $(10,12)$. Del total de las pacientes, 5 requirieron 2 o más intervenciones farmacológicas para el manejo $(6,12$, $13,16)$. No se reportaron características o factores predictores para la respuesta a un tratamiento específico, ni uno de los tratamientos como manejo de elección en el contexto de la gestación. Finalmente, en dos pacientes se reportó el requerimiento de transfusión de hemoderivados prenatal $(6,10)$.

No se encuentra información sobre el uso en el embarazo de medicamentos utilizados en pacientes no gestantes, tales como el danazol -del cual se describió posible asociación con efectos androgénicos en el feto femenino, hipertrofia de clítoris, defectos del seno urogenital, atresia vaginal y genitales ambiguos (21)-, y la ciclofosfamida, que es categoría D de la FDA (22).

En la literatura no hay información que sustente el parto por vía abdominal para las pacientes con 
SE, excepto para las que tienen una indicación obstétrica. En la revisión de los casos, sin embargo, se encuentran los siguientes desenlaces: cinco gestaciones llegaron a término $(9,12,13,15,16)$, ninguna más allá de la semana 39. Cuatro de estos nacimientos fueron vaginales $(9,13,15,16)$ y uno por cesárea de emergencia (12); no se presentaron desenlaces fatales para los fetos-recién nacidos en esos casos. Los demás fueron nacimientos pretérmino $(6,10,11,14,15)$, tres de estos fueron por cesárea de emergencia $(6,14)$.

Pronóstico. El curso clínico de la enfermedad se caracteriza por ser crónico y con recaídas, como en el caso de nuestra paciente.

Respecto a complicaciones específicas, en tres pacientes se reportó el diagnóstico de trastornos hipertensivos asociados al embarazo, que incluyó dos síndromes HELLP $(6,12)$ y una preeclampsia sin criterios de severidad (11). En ninguno de los casos reportados parece haberse presentado neutropenia al momento del diagnóstico, que sí es común en niños y adultos $(2,4)$. En dos pacientes se reportó hemorragia posparto con requerimiento de transfusión de hemoderivados, uno fue un nacimiento vaginal y el otro por cesárea $(14,15)$.

En cuanto al pronóstico perinatal se reportaron dos muertes fetales $(11,15)$. En ambos casos se demostró paso transplacentario de anticuerpos con hemólisis masiva y muerte fetal in utero, por lo que consideramos que podría estar indicada la búsqueda activa de anemia fetal en este tipo de pacientes como en otros de riesgo. Puesto que el estudio de la velocidad pico sistólica mediante doppler de arteria cerebral media es el método no invasivo de elección debido a su alta sensibilidad y especificidad para los casos de anemia severa, consideramos que puede ser una estrategia para el diagnóstico y la usamos en nuestra paciente $(23,24)$.

\section{CONCLUSIONES}

El síndrome de Evans durante la gestación es una entidad rara y su diagnóstico se realiza con pruebas de laboratorio. Puede tener un curso variable du- rante el embarazo, desde una gestación sin mayores alteraciones, como el caso mostrado, o relacionarse con complicaciones obstétricas como el desarrollo de trastornos hipertensivos del embarazo, abruptio placentae y hemorragia posparto. Se puede presentar compromiso fetal, pero no es frecuente. Se dispone de alternativas terapéuticas que incluyen el uso de corticoides, gamaglobulina intravenosa y, en algunos casos, el manejo quirúrgico con esplenectomía, pero aún faltan estudios respecto al tratamiento y pronóstico de la enfermedad que permitan guiar su manejo.

\section{REFERENCIAS}

1. Evans RS, Takahashi K, Duane RT, Payne R, Liu C. Primary thrombocytopenic purpura and acquired hemolytic anemia; evidence for a common etiology. AMA Arch Intern Med. 1951;87:48-65.

2. Michel M, Chanet V, Dechartres A, Morin AS, Piette JC, Cirasino L, et al. The spectrum of Evans syndrome in adults: new insight into the disease based on the analysis of 68 cases. Blood. 2009;114:3167-72.

3. Teachey DT, Manno CS, Axsom KM, Andrews T, Choi JK, Greenbaum BH, et al. Unmasking Evans syndrome: T-cell phenotype and apoptotic response reveal autoimmune lymphoproliferative syndrome (ALPS). Blood. 2005;105:2443-8.

4. Barros MM, Blajchman MA, Bordin JO. Warm autoimmune hemolytic anemia: recent progress in understanding the immunobiology and the treatment. Transfus Med Rev. 2010;24(3):195-210.

5. Mathew P, Chen G, Wang W. Evans syndrome: results of a national survey. J Pediatr Hematol Oncol. 1997;19(5):433-7.

6. Lefkou E, Nelson-Piercy C, Hunt BJ. Evans' syndrome in pregnancy: a systematic literature review and two new cases. Eur J Obstet Gynecol Reprod Biol. 2010;149(1):10-7.

7. Gehrs BC, Friedberg RC. Autoimmune hemolytic anemia. Am J Hematol. 2002;69:258-71.

8. Martin JN, Jr., Bailey AP, Rehberg JF, Owens MT, Keiser SD, May WL. Thrombotic thrombocytopenic purpura in 166 pregnancies: 1955-2006. Am J Obstet Gynecol. 2008;199:98-104.

9. Silverstein MN, Aaro LA, Kempers RD. Evans' syndrome and pregnancy. Am J Med Sci. 1966;252:206-11. 
10. Sherke RR, Rao MS. Anaesthetic management of splenectomy in Evan's syndrome during pregnancy with pregnancy induced hypertension. J Postgrad Med. 2001;47:196-8.

11. Phupong V, Sareepapong W, Witoonpanich P. Evans syndrome and pregnancy: a case report. Bjog. 2004;111:274-6.

12. Boren T, Reyes C, Montenegro R, Raimer K. A case of Evan's syndrome in pregnancy refractory to primary treatment options. J Matern Fetal Neonatal Med. 2007;20:843-5.

13. Passi GR, Kriplani A, Pati HP, Choudhry VP. Isoimmune hemolysis in an infant due to maternal Evans' syndrome. Indian J Pediatr. 1997;64:893-5.

14. Selcuk Tuncer Z, Buyukasik Y, Demirtas E, Tuncer R, Zarakolu P. Pregnancy complicated by Evan's syndrome. Eur J Obstet Gynecol Reprod Biol. 2001;100:100-1.

15. Letts HW, Kredentser B. Thrombocytopenia, hemolytic anemia, and two pregnancies. Report of a case. Am J Clin Pathol. 1968;49:481-6.

16. Julius U, Patzak A, Schaich M, Ehninger G, Kamin G. [Immune thrombocytopenia, anemia and leukopenia during pregnancy. Successful therapy with extracorporeal immunoadsorption]. Dtsch Med Wochenschr. 1997;122:220-4.

17. Longpré B, Cousineau L, Losito R. Association of Evans' syndrome and pregnancy. Unión Med Can. 1970;99(10):1825-7.
18. Ohtsuki T, Inoue N, Sugihara T, Mannoji M, Yawata Y, Togawa A. [A case of Evans' syndrome: thrombocytopenia improved by artificial abortion]. Rinsho Ketsueki. 1984;25:1814-8.

19. Barsony J, Radnoti G, Varnai F. [Simultaneous occurrence of Evans' syndrome and uteroplacental apoplexia]. Orv Hetil. 1968;109:1991-2.

20. Nause SL, Spiegler J, Weichert J, Hartge DR. Therapeutic management of evans syndrome in a pregnancy with maternal systemic lupus erythematosus. Z Geburtshilfe Neonatol. 2015;219:190-2.

21. Brunskill PJ. The effects of fetal exposure to danazol. Br J Obstet Gynaecol. 1992;99:212-5.

22. Zemlickis D, Lishner M, Erlich R, Koren G. Teratogenicity and carcinogenicity in a twin exposed in utero to cyclophosphamide. Teratog Carcinog Mutagen. 1993;13:139-43.

23. Mari G, Norton ME, Stone J, Berghella V, Sciscione AC, Tate D, et al. Society for Maternal-Fetal Medicine (SMFM) Clinical Guideline \# 8: the fetus at risk for anemia-diagnosis and management. Am J Obstet Gynecol. 2015;212:697-710.

24. Mari G, Deter RL, Carpenter RL, Rahman F, Zimmerman R, Moise KJ, Jr., et al. Noninvasive diagnosis by Doppler ultrasonography of fetal anemia due to maternal red-cell alloimmunization. Collaborative Group for Doppler Assessment of the Blood Velocity in Anemic Fetuses. N Engl J Med. 2000;342:9-14. 